\title{
Improved accuracy and robustness of NAA results in a large throughput laboratory by systematic evaluation of internal quality control data
}

\author{
M. J. J. Koster-Ammerlaan, * P. Bode \\ Delft University of Technology, Delft, The Netherlands \\ *Reactor Institute Delft, Mekelweg 15, 2629JB Delft, The Netherlands
}

(Received July 10, 2008)

\begin{abstract}
The laboratory for INAA in Delft processes several thousands of samples per year for multielement determinations in a variety of matrices. Samples are measured on different spectrometers (using well-type and coaxial detectors), by different persons and using many different analytical protocols. All these results should be consistent with each other with respect to the degree of accuracy, i.e., the combination of trueness and precision. A rigorous internal quality control program has been implemented with automatic on-line evaluation. Annually an additional evaluation of the internal quality control results is carried out using statistical techniques. The Naji-plot approach has proven to be an important graphical tool since it provides direct insight in both trueness and precision. It is demonstrated that the degree of accuracy of the results obtained under the large variety of operational parameters is under constant improvement.
\end{abstract}

\section{Introduction}

The laboratory for INAA in Delft processes several thousands of samples per year for multi-element determinations in a variety of matrices. The activities are covered, since the early 1990 s, by a quality management system and an ISO/IEC17025:2005 laboratory accreditation. ${ }^{1,2}$ Any sample analyzed, irrespective of batch size (max. 14 samples) is processed together with internal quality control samples. The laboratory prefers the use of (certified) reference materials for internal quality control; an overview of the variety and frequency of these materials applied in recent years is given in Table 1. A blank capsule is also always included in each batch as an additional quality control sample.

The final results are obtained by holistic analysis in which a simultaneous interpretation is done of the raw data of all (i.e., sometimes 1, sometimes 2-3) measurements ${ }^{3}$ taking into account all peaks including escape peaks. The zeta $(\zeta)$ score is calculated following:

$$
\zeta=\frac{x_{l a b}-x_{r e f}}{\sqrt{u_{l a b}^{2}+u_{r e f}^{2}}}
$$

in which $x_{l a b}=$ value (mass fraction) of the measurement result of the element in the control sample, $x_{r e f}=$ certified mass fraction, $u_{l a b}=$ combined standard uncertainty of the measurement result, $u_{r e f}=$ combined standard uncertainty of the certified value.

The software automatically generates warning flags in the reports if the results of the internal quality control samples violate the warning and action limits of $\zeta>2$ and $\zeta 3$, respectively. Based on intended fitness for purpose, if $>3$ a non-conformity is registered, a root cause analysis is initiated and, if applicable, remedial and corrective actions are implemented, all in compliance with ISO/IEC 17025 clauses 4.9 and 4.11. In addition, quality control charts are generated for inspection of trends. ${ }^{4}$

A large database is thus created with information on results of many quality control materials obtained by several operators (in 2007: 5 persons), at 7 gamma-ray spectrometers ( 3 well-type detectors, 2 coaxial detectors and 2 detectors at the fast rabbit systems for short halflife NAA), in different geometries and under many different analytical protocols, i.e., the combination of sample mass, irradiation, decay and counting times.

Obviously, results obtained by different operators, under different analytical protocols, using different detectors (and counting geometries) and on the basis of different peaks should be consistent with each other with respect to the degree of accuracy, i.e., the combination of trueness and precision. The $\zeta$ criterion provides only a ratio of these characteristics with implicit weakness that a combination of poor degree of both trueness and precision (due to large uncertainty of measurement) may still result in an acceptable $\zeta$ value though providing a false indication of the degree of accuracy.

The components of the $\zeta$ score can, however, by simple rearrangement be used for generation of a different control chart which provides the required insight in degree of accuracy as a function of trueness and precision: the Naji-plot ${ }^{5}$ (Fig. 1). 
The rearrangement of variables is based on the definition of an acceptance criterion $C$, resulting in:

$$
\begin{aligned}
& \zeta=\frac{x_{l a b}-x_{r e f}}{\sqrt{u_{l a b}^{2}+u_{r e f}^{2}}}<C \\
& {\left[\frac{1}{C^{2}} \frac{\left(x_{l a b}^{2}-x_{r e f}^{2}\right.}{u_{r e f}^{2}}\right]-1<\left(\frac{u_{l a b}}{u_{r e f}}\right)^{2}} \\
& \frac{Z^{2}}{C^{2}}-1<\left(\frac{u_{l a b}}{u_{r e f}}\right)^{2}
\end{aligned}
$$

The $Z$-value, $\left(x_{l a b}-x_{r e f}\right) / u_{r e f}$, is a measure of the trueness; the ratio $u_{l a b} / u_{r e f}$ is a measure of the precision of the measurement procedure. Plotting $Z$ against $\left(u_{\text {lab }} / u_{\text {ref }}\right)^{2}$ results in a graph as shown in Fig. 1, in which 'equal acceptance' criteria boundaries are drawn for values of $C$. Moreover, as suggested by ROBOUCH et al., ${ }^{5}$ a maximum value of the ratio $\left(u_{\text {lab }} / u_{\text {ref }}\right)^{2}$ may be defined on basis of fitness for intended purpose.

Table 1. Reference materials measured in the period September 1, 2005 -September 1, 2006

\begin{tabular}{llcllc}
\hline $\begin{array}{c}\text { Reference } \\
\text { material }\end{array}$ & $\begin{array}{l}\text { Name of the } \\
\text { reference material }\end{array}$ & $\begin{array}{c}\text { Number of } \\
\text { measurements }\end{array}$ & $\begin{array}{c}\text { Reference } \\
\text { material }\end{array}$ & $\begin{array}{c}\text { Name of the } \\
\text { reference material }\end{array}$ & $\begin{array}{c}\text { Number of } \\
\text { measurements }\end{array}$ \\
\hline AC-E & $\begin{array}{l}\text { Granite } \\
\text { (IWG-GIT) }\end{array}$ & 5 & $\begin{array}{c}\text { NIST-1577b } \\
\text { (new bottle) }\end{array}$ & Bovine Liver & 34 \\
BCR-186-1 & Pig Kidney & 2 & $\begin{array}{l}\text { NIST-1632C } \\
\text { NIST-1633b }\end{array}$ & $\begin{array}{l}\text { Trace elements in Coal } \\
\text { Coal Fly Ash }\end{array}$ & 56 \\
& $\begin{array}{l}\text { Dogfish Muscle } \\
\text { and Liver }\end{array}$ & 12 & NIST-2709 & San Joaquin Soil & 5 \\
DR-N & Diorite (ANRT) & 2 & NIST-2710 & Montana Soil & 6 \\
GBW07601 & Human Hair & 95 & NIST-2711 & Montana Soil, & 2 \\
GBW09101 & Human Hair & 29 & & Moderately elevated & 3 \\
& & & concentrations & \\
IAEA-085 & Human Hair & 2 & qlo-1 & Quartz Latite (USGS) & 2 \\
IMEP-10 & Polyethylene & 1 & RGM-1 & Rhyolite (USGS) & 2 \\
IMEP-20 & Tuna Fish & 10 & SCo-1 & Cody Shale (USGS) & 2 \\
INCT-TL-1 & Tea Leaves & 85 & SMELS II & Nepheline Syenite & 5 \\
NIST-1515 & Apple Leaves & 1 & STM-1 & (USGS) & 5 \\
& & & & Cd in plastic & 3 \\
NIST-1547 & Peach Leaves & 1 & VDA001 & Cd in plastic & 4 \\
NIST-1577b & Bovine Liver & 67 & VDA002 & & \\
\hline
\end{tabular}

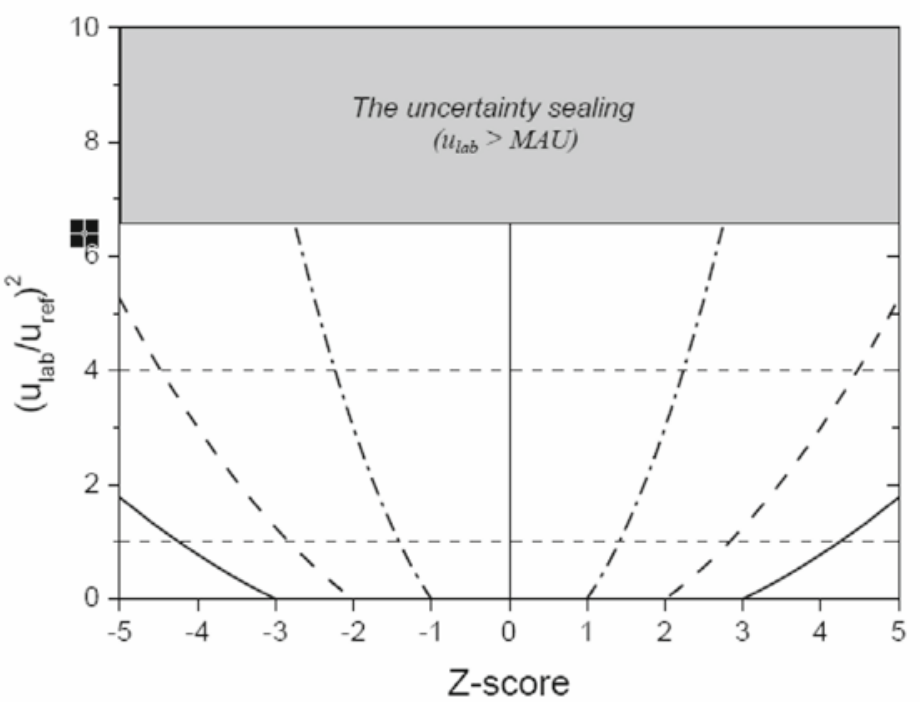

Fig. 1. Naji-plot with four $Z$-regions, $|Z| \leq 1,1<|Z| \leq 2,2<|Z| \leq 3$ and $|Z|>3$ and the Maximum Acceptable laboratory Uncertainty (MAU) 
The Naji plot somehow resembles a basket. Plotting data in this type of graph is indeed almost like putting objects in a basket: the more the data concentrate around $Z=0,\left(u_{l a b} / u_{r e f}\right)^{2}=0$, the higher the degree of accuracy.

It should be noted that the original publication ${ }^{5}$ may be slightly confusing since the definition of the En-score was confounded with the definition of the $\zeta$-score.

The laboratory for INAA in Delft practices the Naji plot in their annual statistical evaluation of all results of the internal quality control samples. Examples of such a statistical evaluation are given in the following paragraphs.

\section{Experimental}

Both a chi-squared test and a Kolmogorov-Smirnov test are applied for assessment of the normality of the distribution of the data if more than 20 measurement results have been obtained for a given element in a given quality control material. Also the standard deviation of the mean, $\mathrm{SEM}_{\text {ext }}$ is compared with the precision of the individual measurement, $\mathrm{SEM}_{\mathrm{int}}$, by calculating

$$
\mathrm{SEM}_{\mathrm{ext}}=\sqrt{\frac{1}{N(N-1)} \cdot \sum_{i=1}^{N}\left(x_{l a b}-\bar{x}\right)^{2}}
$$

and

$$
\frac{1}{\mathrm{SEM}_{\mathrm{int}}}=\sqrt{\sum_{i=1}^{N} \frac{1}{s_{i}^{2}}}
$$

which for almost identical values of $s_{i}$ results in

$$
\mathrm{SEM}_{\mathrm{int}}=\frac{s}{\sqrt{N}} .
$$

If the uncertainty of measurements does not cover all sources of variation, $\mathrm{SEM}_{\text {ext }}$ will be larger than $\mathrm{SEM}_{\text {int }}$.
Such additional sources of variation may be due to, e.g., human factors or mismatch in calibration factors. Since the data used is obtained from measurements on different samples and under different protocols, it may happen that $\mathrm{SEM}_{\text {ext }}<\mathrm{SEM}_{\mathrm{int}}$; in that case apparently the individual truenesses do not vary much from one another.

The database of quality control material analysis results allows for investigating the accuracy of one or all element(s) in each quality control material as well as one or all element(s) in all quality control materials. Naji plots were created for these options, using Microsoft EXCEL.

\section{Results and discussion}

The chi-squared test and the Kolmogorov-Smirnov indicate that not all analysis results of 2006 are normally distributed. An example is given in Table 2. The main cause of non-normality is a mismatch of calibration factors - based on energy efficiency and coincidence summing correction - for different detectors. An additional Dixon Q-, F- and t-test have been applied to the data obtained on 2 individual detectors to investigate outliers and any significant differences in variance and/or the two distributions. Results are given in Table 3 and Table 4, respectively. The differences could be explained by a small mismatch of calibration factors.

An example of comparison of $\mathrm{SEM}_{\text {int }}$ and $\mathrm{SEM}_{\text {ext }}$ is given in Table 5. There are no indications for sources of variation not being covered by the uncertainty of measurement, except for Th. The deviation for this element is not fully understood yet and is now subject of a deeper root cause analysis.

Table 2. Results of chi-squared test for different elements in reference materials

\begin{tabular}{lccl}
\hline Reference material & Element & $\mathrm{n}$ & \multicolumn{1}{c}{ Result test } \\
\hline NIST-1632C & $\mathrm{As}$ & 53 & No normal distribution \\
NIST-1632C & $\mathrm{Br}$ & 55 & Normal distribution \\
NIST-1632C & $\mathrm{Ca}$ & 52 & Normal distribution \\
NIST-1632C & $\mathrm{Co}$ & 55 & No normal distribution \\
NIST-1632C & $\mathrm{Cr}$ & 55 & No normal distribution \\
NIST-1632C & $\mathrm{Fe}$ & 55 & Normal distribution \\
NIST-1632C & $\mathrm{K}$ & 53 & Normal distribution \\
NIST-1632C & $\mathrm{Rb}$ & 54 & Normal distribution \\
NIST-1632C & $\mathrm{Sc}$ & 55 & Normal distribution \\
NIST-1632C & $\mathrm{Sm}$ & 55 & No normal distribution \\
NIST-1632C & $\mathrm{Th}$ & 54 & No normal distribution \\
NIST-1632C & $\mathrm{Zn}$ & 53 & Normal distribution \\
INCT-TL-1 & $\mathrm{Cr}$ & 84 & No normal distribution \\
INCT-TL-1 & $\mathrm{Zn}$ & 84 & Normal distribution \\
GBW07601 & $\mathrm{As}$ & 93 & Normal distribution \\
GBW07601 & $\mathrm{Hg}$ & 94 & Normal distribution \\
GBW07601 & $\mathrm{Se}$ & 94 & Normal distribution \\
GBW07601 & $\mathrm{Zn}$ & 94 & No normal distribution \\
\hline
\end{tabular}


Table 3. Results of Kolmogorov-Smirnov test for different elements on basis of different detectors

\begin{tabular}{ccccc}
\hline Reference material & Element & $\mathrm{n}$ & Detector & Result test \\
\hline NIST-1632C & As & 27 & NPT & Normal distribution \\
NIST-1632C & As & 24 & SUR & Normal distribution \\
NIST-1632C & $\mathrm{Cr}$ & 28 & NPT & Normal distribution \\
NIST-1632C & $\mathrm{Cr}$ & 24 & SUR & Normal distribution \\
NIST-1632C & $\mathrm{Sm}$ & 27 & NPT & Normal distribution \\
NIST-1632C & $\mathrm{Sm}$ & 23 & SUR & Normal distribution \\
NIST-1632C & Th & 28 & NPT & Normal distribution \\
NIST-1632C & Th & 24 & SUR & Normal distribution \\
\hline
\end{tabular}

Table 4. Results of F- and t-test for data obtained on two different well-type detectors

\begin{tabular}{ccccccc}
\hline Element & Detector & Detector & Reference material & $\begin{array}{c}\text { Dixon-Q-test } \\
\text { outliers }\end{array}$ & $\begin{array}{c}\text { F-test } \\
(\alpha=0.05)\end{array}$ & $\begin{array}{c}\text { T-test } \\
(\alpha=0.05)\end{array}$ \\
\hline As & NPT & SUR & NIST-1632C & 0 & Not significant & Not significant \\
Cr & NPT & SUR & NIST-1632C & 0 & Significant & - \\
Sm & NPT & SUR & NIST-1632C & 1 & Not significant & Significant \\
Th & NPT & SUR & NIST-1632C & 0 & Significant & - \\
\hline
\end{tabular}

Table 5. Comparisons of $\mathrm{SEM}_{\mathrm{int}}$ and $\mathrm{SEM}_{\mathrm{ext}}$ for SMELS-II

\begin{tabular}{ccccc}
\hline SMELS-II & & & & \\
\hline Element & Group mean, mg/kg & SEM $_{\text {int }}, \%$ & SEM $_{\text {ext }}, \%$ & $\mathrm{n}$ \\
\hline $\mathrm{Zn}$ & $6.68 \mathrm{E}+03$ & 0.91 & 0.79 & 11 \\
$\mathrm{As}$ & $9.61 \mathrm{E}+01$ & 0.89 & 0.74 & 11 \\
$\mathrm{Br}$ & $1.52 \mathrm{E}+02$ & 0.43 & 0.25 & 11 \\
$\mathrm{Mo}$ & $5.11 \mathrm{E}+03$ & 0.80 & 0.83 & 11 \\
$\mathrm{Sb}$ & $1.75 \mathrm{E}+02$ & 0.48 & 0.44 & 11 \\
$\mathrm{Ce}$ & $1.67 \mathrm{E}+04$ & 0.41 & 0.29 & 11 \\
$\mathrm{Pr}$ & $1.27 \mathrm{E}+03$ & 0.79 & 0.42 & 11 \\
$\mathrm{Yb}$ & $1.90 \mathrm{E}+02$ & 0.52 & 0.44 & 11 \\
$\mathrm{Au}$ & $3.97 \mathrm{E}+00$ & 0.32 & 0.27 & 11 \\
$\mathrm{Th}$ & $3.91 \mathrm{E}+03$ & 053 & 0.91 & 11 \\
\hline
\end{tabular}

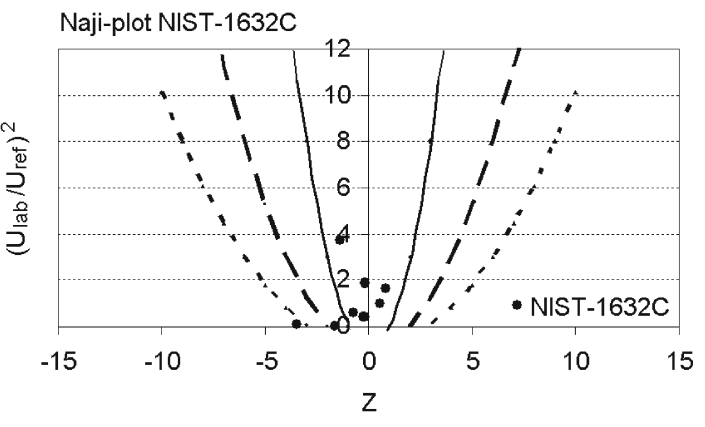

Fig. 2. Naji-plot of certified elements in NIST-1632C

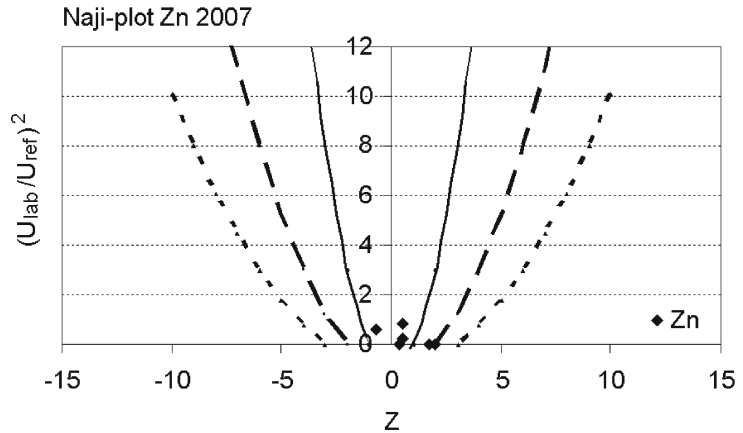

Fig. 3. Naji-plot of zinc in different reference materials 

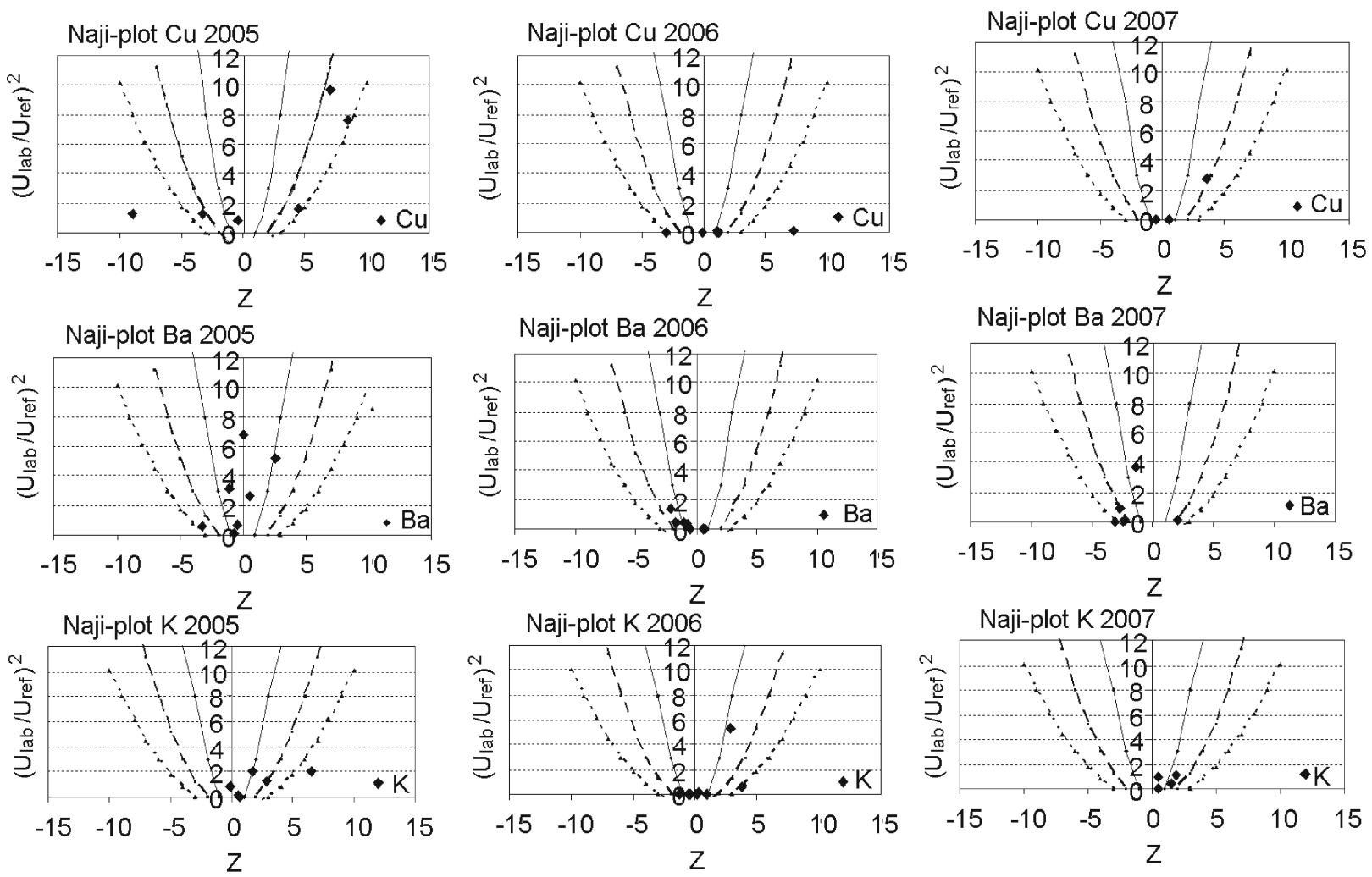

Fig. 4. Naji-plots of $\mathrm{Cu}, \mathrm{Ba}$ and $\mathrm{K}$ in different reference materials based on annual data collected in 3 successive years showing improvement of the degree of accuracy

Examples of Naji-plots are given in Fig. 2 (all certified elements in one reference material) and in Fig. 3 (one element in all reference materials). If data points occur outside the acceptance areas (characterized by $\left.\left(u_{\text {lab }} / u_{r e f}\right)^{2}>6, Z \geq 3\right)$ a root cause analysis procedure is started. In several cases this has led to an identification of the problem and subsequent corrective action and thus to continuous improvement of the degree of accuracy.

This is illustrated by the Naji-plots in Fig. 4, showing the improvement in performance for the elements $\mathrm{K}, \mathrm{Ba}$ and $\mathrm{Cu}$ based on annual data collected in 3 successive years.

For $\mathrm{Cu}$, the main reason for the discrepancies in 2005 could be traced towards the use of the $511 \mathrm{keV}$ in the gamma-ray spectrum of ${ }^{64} \mathrm{Cu}$ which, except for the sometimes also determined gamma-ray spectrum of ${ }^{66} \mathrm{Cu}$ was used for quantification of $\mathrm{Cu}$. The reason for the discrepancies of $\mathrm{Ba}$ and $\mathrm{K}$ was a mismatch of calibration factors between different detectors.

\section{Conclusions}

Naji-plots are a valuable additional tool to common statistical techniques and control charts since they allow for visual inspection of the degree of accuracy (combining trueness and precision) of analysis' results of quality control materials such as certified reference materials. It has resulted at the laboratory for INAA in Delft in further insight in small sources of error, whereas the systematic elimination there of (driven by the quality assurance awareness and laboratory accreditation) contributed to measurable improvements in accuracy and robustness.

\section{References}

1. P. Bode, J. P. VAn Dalen, J. Radioanal. Nucl. Chem., 179 (1994) 141

2. P. Bode, J. Radioanal. Nucl. Chem., 245 (2000) 127.

3. M. BlaAuW, Nucl. Instr. Methods, A353 (1-3) (1994) 269.

4. P. Bode, C. P. VAN DiJK, J. Radioanal. Nucl. Chem., 215 (1997) 87.

5. P. Robouch, Y. Younes, P. Vermaercke, Technical note: PTB-IT-10, 2003, 149, ISSN 0942-1785, ISBN 3-89701-933-3. 